\title{
QUEEN'S
UNIVERSITY
BELFAST
}

\section{A Method for Color Calibration Based on Simulated Annealing Optimization}

Yu, H., Cao, T., Li, B., Dong, R., \& Zhou, H. (2016). A Method for Color Calibration Based on Simulated Annealing Optimization. In 2016 3rd International Conference on Information Science and Control Engineering (ICISCE) (pp. 54-58). [7726119] Institute of Electrical and Electronics Engineers Inc.. https://doi.org/10.1109/ICISCE.2016.22

Published in:

2016 3rd International Conference on Information Science and Control Engineering (ICISCE)

Document Version:

Peer reviewed version

Queen's University Belfast - Research Portal:

Link to publication record in Queen's University Belfast Research Portal

Publisher rights

Copyright IEEE 2016

This work is made available online in accordance with the publisher's policies. Please refer to any applicable terms of use of the publisher.

\section{General rights}

Copyright for the publications made accessible via the Queen's University Belfast Research Portal is retained by the author(s) and / or other copyright owners and it is a condition of accessing these publications that users recognise and abide by the legal requirements associated with these rights.

Take down policy

The Research Portal is Queen's institutional repository that provides access to Queen's research output. Every effort has been made to ensure that content in the Research Portal does not infringe any person's rights, or applicable UK laws. If you discover content in the Research Portal that you believe breaches copyright or violates any law, please contact openaccess@qub.ac.uk. 


\title{
A method for color calibration based on Simulated Annealing Optimization
}

\section{Haifeng Yu, Tian Cao, Huijuan Chen, Dong Rong, Bo Li*, Huiyu Zhou, Minhui Huang}

\begin{abstract}
To accurately reproduce colors, it is necessary to calibrate a digital camera that is used to acquire images. The traditional calibration methods have been used to build a color correction model that has a transformation matrix mapping data from a RGB image to standard XYZ tristimulus. Determination of this matrix is performed through matrix transformation from each color checker patch to its corresponding actual XYZ value, using least-squares regression. However, these methods cannot effectively minimize the CIE DE error because of these algorithms' limitations and CCD dark currents, which can be hard to accurately measure. In this paper, a new color correction model is presented for color calibration and helps minimize the CIEDE2000 error in order to achieve higher color fidelity. Experimental results show that our method significantly outperforms other algorithms.
\end{abstract}

Keywords: color correction model, CCD dark current, CIEDE2000 color difference formula, minimize the mean CIE DE error.

Address all correspondence to: Haifeng Yu, Nanjing University, School of Electronic Science and Engineering, No.163, Xianlin Avenue, Qixia District, Nanjing, Jiangsu Province , China, 210023; Tel: +8615751863731; E-mail:

342032645@qq.com

\section{Introduction}

Achieving a perceptually-consistent color representation is one of the computer vision applications. At present, image color can be affected by many factors such as lighting, viewpoint, and material properties [1]. Due to these factors, the color of the acquired images do not directly reflect - that of the scene. It is necessary to undertake color correction on images in order to improve the accuracy of color reproduction.

Traditional color correction methods can be categorized into two types: the methods based on spectral sensitivity functions and the methods based on color targets (e.g., color checker). The former requires to analyze spectral sensitivity functions to correct the color of images. The camera spectral sensitivities can be measured using a monochromator that generates narrow-band lights and a spectrophotometer that measures its spectral power distributions [2]. On the contrary, the latter is simple but practical. It utilizes a set of color samples of color RGB triplets and the corresponding color XYZ tristimulus values to generate a color correction model. Different methods, including Look-up Table methods and Least-squares Polynomial Regression, White Point Preserving Least Squares and Pattern Search Optimization Methods can be used to create a model. In general, the Look-up Table method shown in [3,4] provides higher accuracy than the other methods, but it is too slow to build robust tables. Polynomial regression [5,6] uses polynomials to establish the mapping relationship and calculate the correction factor using a least squares method. However, this method can only be applied to a range of corrections and may magnify the noise, thus reduces the correction accuracy. Given white color reproduction is an important indicator in color calibration, a constraint for least squares is necessary. This is achieved using white point preserving least squares $[7,8]$. Although this method could assure white accurate reproduction, the accuracy in correcting other colors will be further lowered. The experiment proves that the Pattern Search Optimization method can minimize the errors existing in color calibration and further improve calibration accuracy $[9,10]$. However, one of the concerns is that the initial value is easy to fall into a local optimal solution. However, all of the above methods do not take into account the errors caused by CCD dark current.

In this paper, we combine the CIEDE2000 color difference formula and polynomial regression to form a color correction model. This method is to estimate a matrix M, which maps RGB data to the corresponding XYZ values namely "theoretical standard XYZ values", and then minimizes the CIE DE differences between the standard and theoretical XYZ values to calculate the calibration matrix. Because standard XYZ tristimulus values of colorful targets are difficult to obtain, we use the data obtained from 
the BabelColor Company [11]. However, the brightness of images may change in the calibration process, as the brightness is different from the standard image of the color checker. Consequently, we propose a brightness constraint condition for color calibration. Furthermore, to further reduce the impact of CCD dark current on the color correction, which introduces error into the calibration, we have to deal with CCD dark current.

In order to calculate the calibration matrix, we establish an objective function to minimize the CIEDE2000 color error between the standard and theoretical XYZ tristimulus. For nonlinear optimization, we choose a simulated annealing optimization algorithm to minimize the objective function. Roughly speaking, there are two steps in the proposed color calibration method: (1) establishing a mapping relationship from RGB to XYZ by a $3 \times 3$ matrix using the polynomial regression method to obtain the theoretical XYZ values corresponding to the reference color patches, and (2) minimizing the error between the standard and theoretical XYZ values of the reference color patches by applying the simulated annealing optimization algorithm to calculate the color correction matrix $\mathrm{M}$. We perform experiments using a set of pictures which are captured by a camera. The experimental results indicate that the proposed model achieves better performance in color calibration than several standard methods.

\section{The proposed color calibration model}

To achieve high accuracy of color reproduction, we target at a matrix $\mathrm{M}$ that can minimize the difference between the standard and theoretical XYZ values. Traditional methods apply CIELAB $\Delta E_{76}$ to measure the difference. However, such measurement cannot conform to the chromatic induction mechanisms of the human visual system. Thus, we utilize the CIEDE2000 color difference in the proposed model.

In the following subsections, firstly we introduce the method to convert color values from RGB to $\mathrm{XYZ}$ in section 2.1. The method to eliminate the influence of CCD dark current is then introduced in section 2.2. The optimization method for calculating the correction matrix is finally introduced in section 2.3 .

\subsection{Converting color values from RGB to $X Y Z$}

As mentioned above, we establish a relationship that the RGB values can be mapped to XYZ values by a $3 \times 3$ matrix using the polynomial regression method. For concreteness, we denote $Q=[X, Y, Z]$ as a $q \times 3$ matrix that can be made up of theoretical color XYZ tristimulus values, where $q$ is the number of the color targets. Similarly, let the matrix $P=[B, G, R]$ represent measured color RGB triplets. Roughly speaking, $P$ represents the values of the color checker obtained from a picture and $Q$ denotes the theoretical values. Through linear mapping, we can search for a $3 \times 3$ matrix $M$ that meets this mapping relationship:

$$
[\mathrm{X}, \mathrm{Y}, \mathrm{Z}]=[\mathrm{B}, \mathrm{G}, \mathrm{R}] M
$$

The transformation method is generally based on a polynomial model. To enable the matrix $\mathrm{M}$ denoted in equation (1) to obtain the perceptually accurate mapping relationship, we intend to reduce the errors calculated in terms of perceptual difference. We then calculate the CIE DE difference between the standard XYZ tristimulus and theoretical values. The calibration result of the CIE DE differences should conform to the chromatic induction mechanisms of the human visual system. The traditional methods measure color difference based on the CIELAB color space. However, because of its own limitations, for instance non-uniformity, we utilize CIEDE2000 color difference formula as the measurement of color difference in the proposed model. The CIEDE2000 color-difference is based on the CIELAB color space 
and is proved to fit best to the characteristics of the human vision system.

\subsection{Eliminating the influence of CCD dark current}

The values of CCD dark current are not related to the intensity of illumination, which are influenced by the material properties of CCD, environmental temperature and others. The image intensity for R, G and B channels contains additive interference of CCD dark current, however, the traditional calibration methods generally ignore the influence of CCD dark current. Furthermore, the imaging environment of a digital scene is complex and changeable, thus CCD dark current makes a relatively larger impact on imaging quality in many cases. In order to obtain images of high quality, CCD dark current is to be processed.

Taking into account the previous conversion expressions, we can redefine the color correction model as a function of eliminating the influence of CCD dark current:

$$
[\mathrm{X}, \mathrm{Y}, \mathrm{Z}]=\left[\left(B-\tau_{b}\right),\left(G-\tau_{g}\right),\left(R-\tau_{r}\right)\right] M,
$$

Where $\tau_{b}, \tau_{g}, \tau_{r}$ are CCD dark current values for B,G and R channels respectively.

\subsection{Color error optimization using Simulated Annealing}

In this section we report the Simulated Annealing Optimization method for color calibration. It has been developed to improve the accuracy of color calibration.

Let $\bar{Q}$ be a $q \times 3$ matrix containing the standard XYZ tristimulus values, and $Q$ be a $q \times 3$ matrix containing theoretical XYZ values calculated from equation (2). Then we can find a $3 \times 3$ matrix $M$ to reduce color errors between the standard XYZ tristimulus and theoretical values of the reference color patches. In order to also minimize the maximum errors which we have in color calibration, we have to add the $\|\bar{Q}-\bar{Q}\|_{\infty}$ in the function which we want to minimize. Furthermore, we also want to handle the noise amplification that may occur due to the matrixing process. To keep the amplification low, we add the $\operatorname{stdev}(\bar{Q}, Q)$ into the objective function.

The objective function we want to minimize is:

$$
\eta=\alpha \times\left(\frac{\sum_{i=1}^{q} \Delta E_{00, i}(\bar{Q}, \bar{Q})}{q}\right)+\beta \times\|\bar{Q}-\bar{Q}\|_{1}+\delta \times\|\bar{Q}-\bar{Q}\|_{\infty}+\mu \times \operatorname{stdev}(\bar{Q}, \bar{Q}),
$$

Where $\alpha, \beta, \delta, \mu$ are weighting factors that determine the relative importance of the terms. We do not have any ranking of the importance of the members in equation (3), so the weighting factors $\alpha, \beta, \delta, \mu$ are set to 1 .

On problem solving of nonlinear optimization, the Simulated Annealing algorithm is one of the best direct search methods, with high practicality in solving practical problems. It has significant advantages compared with the Pattern Search method and Simulated Genetic method, for instance, the global convergence and efficiency. Furthermore, this method not only avoids the overdependence on an initial value but also overcomes the randomness of the simulated genetic algorithm. For a function which doesn't explicitly make use of the derivatives in minimization, we choose the Simulated Annealing algorithm for equation (3). 
In this paper, the XYZ tristimulus of a color checker image is used as a uniform standard. However, the brightness of the image may change during the calibration process.. Consequently, in order to make the brightness of the image consistent, we here propose a brightness constraint condition for color calibration.

We multiply the brightness values of the reference color patches with an adjustment coefficient $\lambda$, where the initial value of $\lambda$ can be set to 1 . As we have known, the XYZ tristimulus values in the CIE $\mathrm{XYZ}$ color space show a linear relationship with the brightness in the CIE xyY color space. Consequently, the matrix $\bar{Q}$ containing the XYZ tristimulus values of the reference color patches is redefined as $\bar{Q}=[\lambda \mathrm{X}, \lambda \mathrm{Y}, \lambda \mathrm{Z}]$. As said above, we need to work out the correction matrix $\mathrm{M}$ using the Simulated Annealing algorithm. The next step is to obtain the theoretical XYZ tristimulus values of the color checker using equation (2), and then to determine whether or not the brightness values converted from the theoretical and original colors satisfies the brightness constraint condition. If not, we then slightly adjust the adjustment coefficient in the negative direction according to a step-length parameter, which can be set on the basis of the actual situation. Finally, we will figure out the correction matrix till the brightness difference of the image between before and after the calibration satisfies the brightness constraint condition.

The constraint is usually related to the white color brightness, which can be written as:

$$
\left|Y_{t}-Y_{m}\right|<\varepsilon,
$$

Where $Y_{t}, Y_{m}$ represent the brightness values of the theoretical and original color in the CIE XYZ color space, respectively. $\varepsilon$ is the maximum allowable for the change of the brightness. In this paper, we set $\varepsilon$ to 5, and eventually the matrix $\mathrm{M}$ obtained from the color correction model can meet the constraint.

\section{Experimental results}

To evaluate the effectiveness of the proposed color calibration based on Simulated Annealing Optimization, we calibrated a digital camera using the Macbeth ColorChecker (24 color patches) or the X-Rite ColorChecker Digital SG (140 color patches). An achromatic image of the color checker is acquired under an unknown light source. The RGB values across each patch are averaged to make it more representative, resulting in the set of color samples. As the CCD dark current are widespread to all the patches, the CCD dark current needs to be removed from this dataset.

As the light source is located at the top of the light booth, it is inevitable that there are some variations in the illumination irradiance across the color checker that was placed on the floor of the light booth. Although this non-uniformity is barely visible in the images of the color checker, it may magnify the noise in the color calibration. Thus the true XYZ coordinates of the reference color patches may be difficult to obtain accurately in that environment with an unknown light source.

Based on the measured background, we scale the RGB values of each patch from the image of the color checker to simulate the higher color fidelity. Thus, we have an original image captured under unknown lighting and one that represents the image that would have been captured in that environment. The set of these colors, represented by vectors $P$, will be used as the original data. And the set of "true" tristimulus values of the color patches is $\bar{Q}$, which is provided by the BabelColor Company.

To confirm the performance of our simulated annealing algorithm (referred to as SA), we perform a set of tests to compare our SA algorithm with other three methods: least-squares polynomial regression (LS), white point preserving least squares (WPPLS) and pattern search optimization method (PS). In the experiments, the step-length parameter is 0.01 , the correction matrix $\mathrm{M}$ is initialized to be a unit matrix, $\varepsilon=5$ and $\lambda=1$. The quantitative measure of each color correction algorithm's performance is the color error based on the CIEDE2000 color difference formula. 
Table 1 Differences among these color correction methods, computed using the corrected images, respectively.

\begin{tabular}{cllllll}
\hline & Min & Max & Mean & Median & St.dev & \% $\Delta \mathbf{E}<3$ \\
\hline WPPLS & 1.2811 & 16.4911 & 8.2232 & 7.9495 & 4.7520 & 29.2 \\
LS & 0.2852 & 13.7679 & 4.8207 & 4.915 & 3.2735 & 33.4 \\
PS & 0.4949 & 10.8388 & 4.6748 & 4.3689 & 3.1526 & 37.5 \\
SA & 0.1674 & 9.5275 & 3.9893 & 3.3349 & 2.4991 & 45.8 \\
\hline
\end{tabular}

All the methods have been evaluated, as shown in Table 1, in terms of CIEDE2000 color difference, using the Macbeth ColorChecker. Table 1 gives the statistics of multiple calibration methods. The mean errors of the SA method are smaller than any of the other corresponding methods. For a more accurate comparison between these methods, we also evaluate the standard deviations for the patches applying the correction matrix estimated by the four methods. As seen in Table 1, the standard deviation of our method is lower than those of the other three methods.

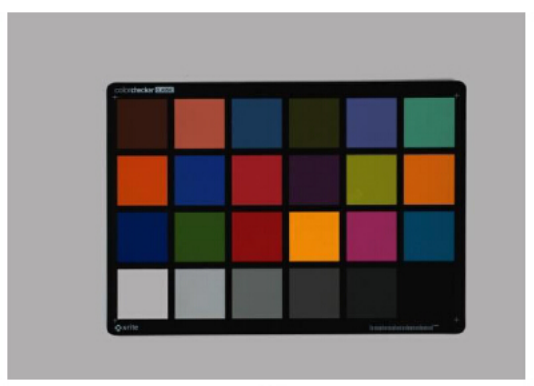

(a)

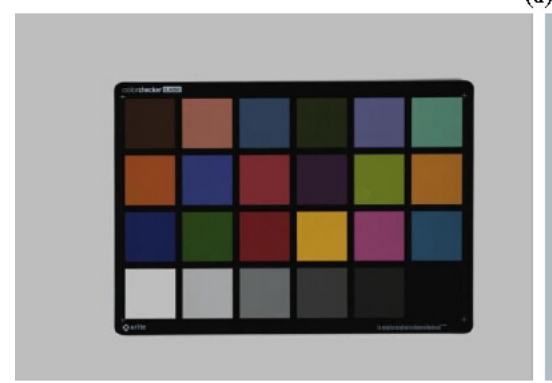

(b)

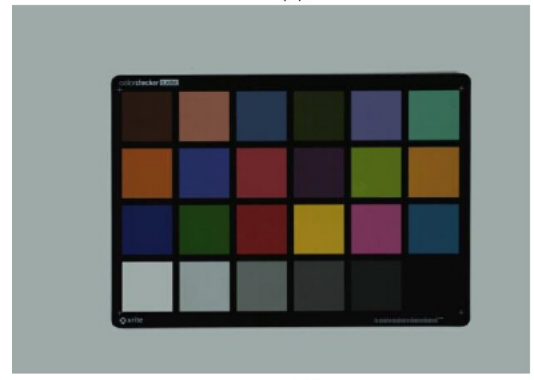

(d)

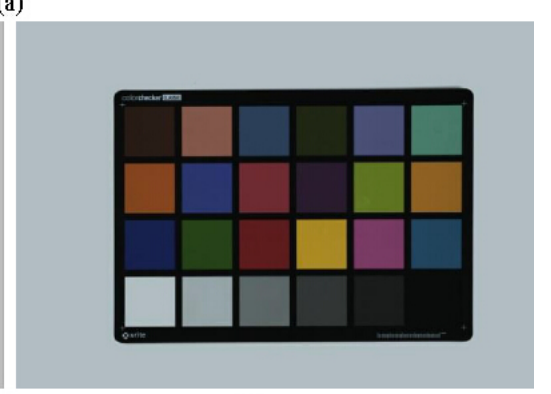

(c)

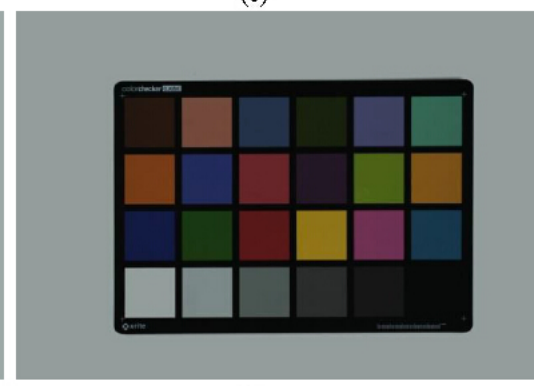

(e)

Fig. 1 Comparative corrected images of four correction methods: (a) the image captured by a digital camera under an unknown uniform illuminant, (b) our color calibration algorithm, (c) PS method, (d) LS method and (e) WPPLS method.

Fig. 1 shows the performance of the various color correction algorithms on an example image captured using an digital camera under an unknown illuminant. We observe that the image in Fig. 1(b) processed using the proposed color correction method shows the highest colorimetric accuracy.

Table $2 \Delta E_{00}$ statistics for our method by eliminating dark current or not.

\begin{tabular}{lllllll}
\hline & Min & Max & Mean & Median & St.dev & \% $\Delta \mathbf{E}<3$ \\
\hline not eliminate & 0.3586 & 11.2721 & 4.4673 & 3.7347 & 2.6752 & 42.7 \\
eliminate dark current & 0.1764 & 9.8342 & 4.1375 & 3.4657 & 2.5283 & 45.8 \\
\hline
\end{tabular}

To evaluate how CCD dark current across the color checker affects the calibration results, we perform a number of tests which are shown in Tables 2. The results demonstrate that our proposed 
calibration techniques is less likely to be affected by CCD dark current.

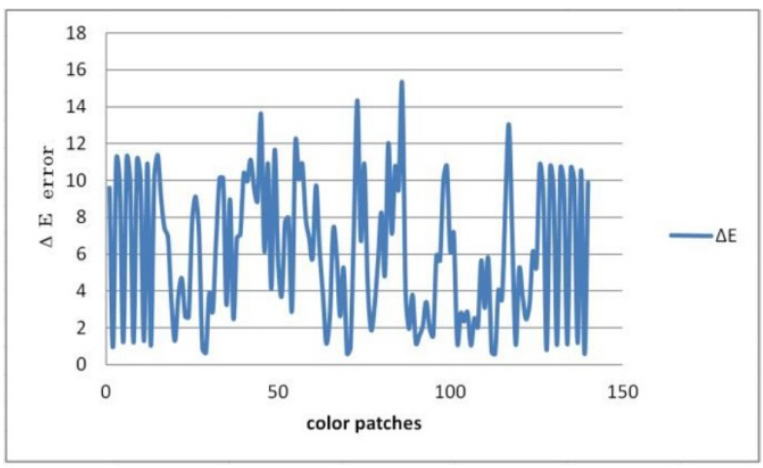

(a)

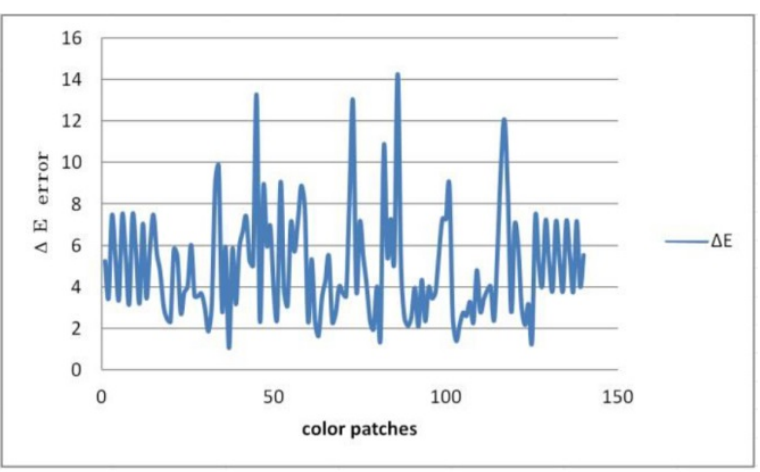

(b)

Fig. 2 Statistics for test set: (a) the statistics using the Macbeth ColorChecker for training set and X-Rite ColorChecker Digital SG for test set, (b) the statistics using the X-Rite ColorChecker Digital SG for both training and test set.

We have also investigated the robustness of the SA solution by increasing the cardinality of the color patches. In this test, we firstly estimate the calibration matrix based on the Macbeth ColorChecker and the X-Rite ColorChecker Digital SG, respectively. Then we apply the calibration matrix to the test set to obtain the statistics for the $\Delta E_{00}$, where the test set is X-Rite ColorChecker Digital SG. In Fig. 2, we report the statistics for the values of $\Delta E_{00}$ obtained on the test sets. As seen in Fig. 2, Fig. 2(b) has the lower mean value than that of Figs. 2(a). It seems that the greater cardinality of the color patches is, the better robustness of the algorithm will be. Overall, we have confirmed that the proposed method has a high accuracy of the color reproduction.

\section{Conclusion}

In this paper we proposed a color calibration model that does not require uniform irradiance across the color targets. Thus it can avoid the dependence on the irradiance compared with the other methods. Besides, it also does not require an objective function to minimize differentiable. More importantly, the test images can be captured from digital cameras with unknown illumination, and the colors of the resulting images can still be well reproduced.

The tests show that CCD dark current can significantly affect the resulting correction matrix and reduce the color reproduction accuracy. The proposed calibration method was slightly less accurate than the traditional methods in the situations where CCD dark current can be controlled. Furthermore, the experiment results show that our model does not change the brightness of the resulting images, and has better noise immunity and high accuracy of the color reproduction.

\section{Acknowledgments}

This work was supported by the National Natural Science Foundation of China (Grant No.61401239).

\section{References}

1. Barnard K, Funt B. Camera characterization for color research[J]. Color Research \& Application, 2002, 27(3): 152-163.

2. J. Nakamura. Image Sensors and Signal Processing for Digital Still Cameras. CRC Press, 2006. 4321, 4323

3. Hung P C. Colorimetric calibration in electronic imaging devices using a look-up-table model and interpolations[J]. Journal of Electronic Imaging, 1993, 2(1): 53-61.

4. Kim Y T, Cho Y H, Lee C H, et al. Color look-up table design for gamut mapping and color space conversion[C]//DPP2003: IS\&T's International Conference on Digital Production Printing and Industrial Applications. 2004: 28-29.

5. Mendes L, Carvalho P. Adaptive polynomial regression for colorimetric scanner calibration using genetic algorithms[C]//Intelligent Signal Processing, 2005 IEEE International Workshop on. IEEE, 2005: 22-27. 
6. Xia M, Saber E, Sharma G, et al. End-to-end color printer calibration by total least squares regression[J]. Image Processing, IEEE Transactions on, 1999, 8(5): 700-716.

7. Finlayson G D, Drew M S. Constrained least-squares regression in color spaces[J]. Journal of Electronic Imaging, 1997, 6(4): 484-493.

8. Vrhel M J, Trussell H J. Color correction using principal components[J]. Color Research \& Application, 1992, 17(5): 328-338.

9. Bianco S, Bruna A, Naccari F, et al. Color space transformations for digital photography exploiting information about the illuminant estimation process[J]. JOSA A, 2012, 29(3): 374-384.

10. Bianco S, Gasparini F, Russo A, et al. A new method for RGB to XYZ transformation based on pattern search optimization[J]. Consumer Electronics, IEEE Transactions on, 2007, 53(3): 1020-1028.

11. Pascale D. RGB Coordinates of the Macbeth color checker[J]. The BabelColor Company, 2006: 1-16 